\title{
Opiniones de los alumnos sobre actividades realizadas en una asignatura con docencia inversa. Thoughts of the students about activities performed in a subject with flip teaching.
}

\author{
Ángeles Calduch-Losa ${ }^{1}$, Vicent Blanes-Selva ${ }^{1}$, Fernando Alcina-Sanchis ${ }^{1}$, Vicent Ahuir-Esteve ${ }^{1}$, Manuel Moscoso- \\ García $^{2}$ \\ mcalduch@eio.upv.es, viblasel@inf.upv.es, feralsa5@inf.upv.es, viahes@eui.upv.es, moscoso_man@gva.es \\ ${ }^{1}$ Escola Tècnica Superior d’Enginyeria Informàtica \\ Universitat Politècnica de València \\ València, España \\ ${ }^{2}$ Conselleria de Sanitat \\ Generalitat Valenciana \\ València, España
}

\begin{abstract}
Resumen - En la docencia de una asignatura de primer curso de grado se han aplicado técnicas de clase inversa, pidiendo al alumno: preparar el contenido teórico de la clase con antelación, utilizar la herramienta Kahoot! para revisar los contenidos al finalizar los temas, visionar vídeos relacionas con la asignatura utilizando la plataforma YouTube y comentar noticias de actualidad del ámbito de la materia en un grupo cerrado de Facebook. El objetivo de este trabajo es conocer el punto de vista del alumnado sobre las técnicas de clase inversa mencionadas. Para ello se ha llevado a cabo una encuesta que nos permitirá obtener información sobre las preferencias de los alumnos respecto a estos métodos y su utilidad en el aprendizaje de la asignatura.
\end{abstract}

Palabras clave: Encuesta, alumnos, docencia inversa, innovación docente

Abstract- In the teaching of a first-year university grade course, flip teaching techniques have been used, requiring students to: prepare the theoretical content of the class in advance, use the online tool Kahoot! In order to review the contents when finalising the subjects, visualize videos related to the subject using the YouTube platform and comment current news from the field of the subject in a closed group of Facebook. The objective of this work is to know the students' point of view about the flip teaching techniques mentioned. To achieve this, a survey has been carried out that will allow us to obtain information about the students' preferences regarding these methods and their usefulness of learning the subject.

Keywords: Survey, students, flip teaching, teaching innovation

\section{INTRODUCCIÓN}

El término docencia inversa se acuñó en 2007 en Woodland Park High School, Colorado, aunque con anterioridad se habían desarrollado métodos de enseñanza que son precedentes de elementos de flipped learning (Prieto-Martín, 2017).

Existen diferentes denominaciones que hacen referencia a estos métodos de enseñanza, como son: docencia inversa, clase inversa, flipped learning, docencia invertida, aprendizaje inverso, flip teaching, flipped classroom y aula inversa.

Se entiende por clase inversa la metodología que promueve desplazar la parte teórica y de clase magistral fuera del aula, haciendo uso de las TIC. De este modo, el profesor proporciona el material teórico en formato digital, haciendo uso de soportes tales como vídeos, podcast y documentos (Terrasa-Barrena \& Andreu-García, 2015).

En este contexto, el estudiante debe tomar un papel más activo, realizando previamente las tareas que en el método tradicional llevaba a cabo en el aula. Mientras, el profesor adopta el papel de guía, orientando a los alumnos en la adquisición de sus competencias. Las sesiones de aula se adaptan a las necesidades de los estudiantes, quienes realizan consultas centrándose en las partes del temario en las que han experimentado más dificultades (García-Barrera, 2013).

En este trabajo se presentan las acciones concretas realizadas en una asignatura de primer curso de grado. Además, se realizó una encuesta a los alumnos en la que se les pedía que valoraran en una escala de Likert del 1 al 5 su interés por cada una de las acciones planteadas y su utilidad como parte del aprendizaje.

\section{CONTEXTO}

La experiencia que se explica en este trabajo se ha desarrollado en la Universitat Politècnica de València, en el Grado en Ingeniería Informática que se imparte en la Escola Tècnica Superior d'Enginyeria Informàtica. La asignatura en la que se ha llevado a cabo es Estadística, materia obligatoria de formación básica que se imparte en el cuatrimestre B del primer curso.

El estudio se ha realizado en dos grupos de teoría de aula y seminario. En cada grupo se han impartido dos clases semanales, cada una de ellas de 1,5 horas de duración, que corresponden a 4,5 créditos por grupo. Se ha contado con 1,125 créditos de apoyo en total.

Las acciones realizadas dentro de la metodología indicada han sido las mismas para los dos grupos de alumnos, ambos tuvieron a la misma profesora en las clases de teoría de aula y seminario y se impartieron en la misma lengua. Por lo tanto, se han podido agrupar las respuestas de todos los alumnos, dando lugar a un tamaño muestral de 67 estudiantes. 


\section{A. Necesidad}

La realización de esta encuesta ha surgido de la necesidad de conocer el punto de vista de los alumnos respecto a las acciones realizadas, ya que parece de suma importancia obtener retroalimentación a la hora de evaluar los métodos empleados más allá de la percepción del docente y los resultados académicos obtenidos por los estudiantes.

\section{B. Objetivos}

El objetivo al hacer este estudio es explicar cómo se ha aplicado la metodología flip teaching con estos alumnos y presentar la opinión de los estudiantes respecto a ella, para lo que se les sondeó haciendo uso de una encuesta.

La realización de esta encuesta tiene una doble finalidad, por una parte constatar el grado de satisfacción del alumnado con las acciones realizadas, y por otra, conocer el nivel de utilidad percibido por los encuestados.

\section{Público objetivo}

El presente trabajo pretende ser de utilidad para los docentes que deseen adaptar o mejorar las técnicas de flip teaching, así como animar a hacer uso de esta metodología a aquellos que no la están utilizando.

\section{DESCRIPCIÓN}

Pasamos a concretar las actividades que se han realizado en la asignatura con estos dos grupos de alumnos, y de cómo hemos obtenido su opinión acerca de ellas.

Desde el inicio del curso, la docente ha proporcionado a los alumnos los materiales en formato pdf a través de la intranet de la asignatura. Estos archivos contenían las unidades didácticas correspondientes a los temas de la materia y ejercicios con el desarrollo del problema o en su defecto con los resultados. Así, los estudiantes ya sabían qué documentos tenían que leer antes de cada clase y de este modo, en el aula consultaban las dudas sobre aquellos conceptos que no les habían quedado claros.

Se ha utilizado la herramienta Kahoot!, aplicación web en la que los docentes pueden crear cuestionarios que los alumnos contestan en un tiempo determinado haciendo uso de sus dispositivos electrónicos, obteniendo puntos por cada respuesta correcta y en función del tiempo. Al final se genera un ranking con las puntuaciones de los participantes.

Además, a través del portal web YouTube se ha tenido acceso a vídeos que, de manera ilustrativa, muestran conceptos y términos estadísticos.

Finalmente, se creó un grupo cerrado en la red social Facebook en el que se fomentó la publicación y el intercambio de comentarios sobre noticias actuales con curiosidades y aplicaciones estadísticas.

Para conocer la opinión del alumnado acerca de estas actividades, la última semana de clase se solicitó a los estudiantes que expresaran su grado de conformidad con una serie de afirmaciones, utilizando una escala de Likert. Los alumnos han valorado, por una parte, si les han gustado y les han servido las herramientas utilizadas, y por otra, han indicado su nivel de conformidad con afirmaciones más generales acerca de la metodología flip teaching.
La encuesta que han contestado los 67 estudiantes puede verse en la Tabla 1. Consta de 17 afirmaciones, de las que en 15 de ellas se contesta según una escala de Likert de 5, mientras que en las otras 2 eran opciones de respuestas cerradas.

Tabla 1: Encuesta realizada a los alumnos.

Afirmación
1) Me ha gustado utilizar Kahoot!
2) Kahoot! me ha ayudado a estudiar
3) Me ha gustado ver los vídeos de
YouTube
4) Los vídeos de YouTube me han
ayudado a entender mejor la
asignatura
5) Me ha gustado utilizar el grupo
Facebook
6) El grupo de Facebook me ha
ayudado a ver aplicaciones de la
asignatura
7) Me ha gustado la visita de
profesores extranjeros
8) Leo la teoría antes de las clases
9) Cuando la he leído, me ha
ayudado

10) Si no la he leído, ¿cuál es el motivo? (falta de tiempo, falta de ganas o indicar otro motivo)

11) Me ha gustado ver la asignatura a través de los ejercicios

12) Me gustaría que este tipo de docencia se impartiera en más asignaturas

13) Estoy satisfecho/satisfecha con la metodología utilizada en las clases de aula

14) Con esta metodología en clase, ha mejorado mi visión sobre la asignatura

15) Recomendaría este tipo de docencia a futuros alumnos

16) Las actividades realizadas en las clases inversas me han mostrado utilidades de la asignatura que sólo con teoría no alcanzaría a apreciar

17) Ahora me fijo más en las noticias con algún componente de la asignatura
$1 \square 2 \square 3 \square 4 \square 5 \square$

Sí $\square$ No $\square$ A veces $\square$ $1 \square 2 \square 3 \square 4 \square 5 \square$

Tiempo $\square$ Ganas $\square$ Otros $\square$

$1 \square 2 \square 3 \square 4 \square 5 \square$

$1 \square 2 \square 3 \square 4 \square 5 \square$

$1 \square 2 \square 3 \square 4 \square 5 \square$

$1 \square 2 \square 3 \square 4 \square 5 \square$

$1 \square 2 \square 3 \square 4 \square 5 \square$

$1 \square 2 \square 3 \square 4 \square 5 \square$

$1 \square 2 \square 3 \square 4 \square 5 \square$ 


\section{RESUltados}

Se ha estudiado el impacto de las actividades llevadas a cabo mediante el análisis de la encuesta mostrada en la Tabla 1, utilizando el software estadístico R. Se muestran únicamente los resultados de las afirmaciones más relevantes de este estudio. Las aseveraciones 7 y 17 no tienen relación con la metodología.

Para cada afirmación se presenta la media obtenida, y también se analiza el porcentaje de alumnos que se ha mostrado de acuerdo (4 y 5 en la escala de Likert), neutral (3 en la escala) y en desacuerdo (valores 1 y 2). Los resultados también se presentan mediante un diagrama de barras para algunas de las afirmaciones.

En general, los resultados han sido positivos. Apreciamos cómo en la mayoría de las afirmaciones la media de las respuestas en la escala de Likert es mayor a 3. Consideramos que un resultado mayor que 3,5 es un resultado positivo y negativo si es menor que 2,5.

Respecto a las herramientas de aprendizaje, la preferida por los encuestados ha sido Kahoot!, obteniendo un 4,4 sobre 5 . Sin embargo, su valoración en cuanto a la ayuda al estudio que les ha proporcionado ha sido neutral, con un 3,39 sobre 5 .

Tabla 2: Resultados de la afirmación 1, "Me ha gustado utilizar Kahoot!”

\begin{tabular}{cccc}
\hline Media & Acuerdo & Neutral & Desacuerdo \\
\hline 4,40 & $86,57 \%$ & $8,96 \%$ & $4,47 \%$ \\
\hline
\end{tabular}

Tabla 3: Resultados de la afirmación 2, “Kahoot! me ha ayudado a estudiar"

\begin{tabular}{cccc}
\hline Media & Acuerdo & Neutral & Desacuerdo \\
\hline 3,39 & $50,75 \%$ & $31,34 \%$ & $17,91 \%$ \\
\hline
\end{tabular}

En segundo lugar, están los vídeos de YouTube, que también han sido muy bien valorados, aunque de nuevo opinan que no les han ayudado a entender mejor la asignatura.

Tabla 4: Resultados de la afirmación 3, "Me ha gustado ver los vídeos de YouTube"

\begin{tabular}{cccc}
\hline Media & Acuerdo & Neutral & Desacuerdo \\
\hline 4,15 & $67,16 \%$ & $19,40 \%$ & $13,44 \%$ \\
\hline
\end{tabular}

Tabla 5: Resultados de la afirmación 4, "Los vídeos de YouTube me han ayudado a entender mejor la asignatura”

\begin{tabular}{cccc}
\hline Media & Acuerdo & Neutral & Desacuerdo \\
\hline 3,41 & $41,79 \%$ & $38,81 \%$ & $19,40 \%$ \\
\hline
\end{tabular}

El grupo de Facebook ha sido valorado de una manera neutral, ya que ha recibido unas valoraciones de 2,63 y 2,87 en las dos afirmaciones planteadas.

Tabla 6: Resultados de la afirmación 5, "Me ha gustado utilizar el grupo de Facebook”

\begin{tabular}{cccc}
\hline Media & Acuerdo & Neutral & Desacuerdo \\
\hline 2,63 & $19,40 \%$ & $32,81 \%$ & $47,79 \%$ \\
\hline
\end{tabular}

Tabla 7: Resultados de la afirmación 6, "El grupo de Facebook me ha ayudado a ver aplicaciones de la asignatura”

\begin{tabular}{cccc}
\hline Media & Acuerdo & Neutral & Desacuerdo \\
\hline 2,87 & $28,35 \%$ & $29,85 \%$ & $41,80 \%$ \\
\hline
\end{tabular}

Podemos observar que muy pocos alumnos leen siempre la teoría antes de acudir a las clases, mientras que prácticamente uno de cada tres estudiantes no la leen nunca. Preguntados por los motivos, más de la mitad coinciden en no haber leído la teoría por falta de ganas. Al preguntar a los que habían leído la teoría si esto les ha ayudado, sólo uno de cada cuatro ha dicho que no.

Tabla 8: Resultados de la afirmación 8, "Leo la teoría antes de las clases"

\begin{tabular}{ccc}
\hline Sí & A Veces & No \\
\hline $2,98 \%$ & $65,67 \%$ & $31,35 \%$ \\
\hline
\end{tabular}

Tabla 9: Resultados de la afirmación 9, “Cuando la he leído, me ha ayudado”

\begin{tabular}{cccc}
\hline Media & Acuerdo & Neutral & Desacuerdo \\
\hline 3,47 & $44,78 \%$ & $31,34 \%$ & $23,88 \%$ \\
\hline
\end{tabular}

Tabla 10: Resultados de la pregunta 10, "Si no la he leído, ¿cuál es el motivo?”

\begin{tabular}{cccc}
\hline Tiempo & Ganas & Tiempo y Ganas & Otros \\
\hline $31,25 \%$ & $48,44 \%$ & $9,38 \%$ & $10,93 \%$ \\
\hline
\end{tabular}

Por último, veamos cómo han sido valoradas las afirmaciones relacionadas con la metodología utilizada:

"Me ha gustado ver la asignatura a través de los ejercicios" tiene un 4,07 de valoración media, y sólo un $6 \%$ de los alumnos la han valorado con un 2 , mientras que ninguno de ellos la valoró con un 1.

Tabla 11: Resultados de la afirmación 11, "Me ha gustado ver la asignatura a través de los ejercicios”

\begin{tabular}{cccc}
\hline Media & Acuerdo & Neutral & Desacuerdo \\
\hline 4,07 & $73,13 \%$ & $20,90 \%$ & $5,97 \%$ \\
\hline
\end{tabular}




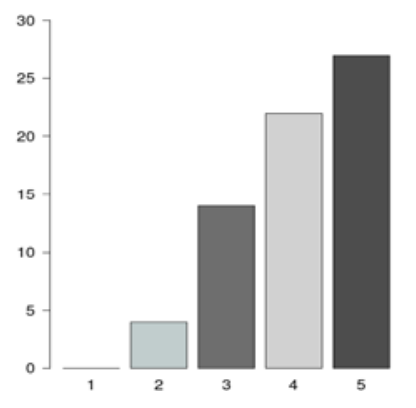

Figura 1: Resultados afirmación 11

El resto de afirmaciones vamos a verlas por orden de valoración:

"Estoy satisfecho/satisfecha con la metodología utilizada en las clases de aula de la asignatura”: sólo hay un $10 \%$ de ellos que dicen que no lo está.

Tabla 12: Resultados de la afirmación 13, “Estoy satisfecho/satisfecha con la metodología utilizada en las clases de aula”

\begin{tabular}{cccc}
\hline Media & Acuerdo & Neutral & Desacuerdo \\
\hline 3,94 & $71,64 \%$ & $17,91 \%$ & $10,45 \%$ \\
\hline
\end{tabular}

"Me gustaría que este tipo de docencia se impartiera en otras asignaturas": aunque el porcentaje de estudiantes que no está de acuerdo es menor a la anterior afirmación, el número de estudiantes en la parte neutral es mucho mayor.

Tabla 13: Resultados de la afirmación 12, “Me gustaría que este tipo de docencia se impartiera en más asignaturas"

\begin{tabular}{cccc}
\hline Media & Acuerdo & Neutral & Desacuerdo \\
\hline 3,89 & $64,18 \%$ & $23,88 \%$ & $8,94 \%$ \\
\hline
\end{tabular}

"Recomendaría este tipo de docencia a futuros alumnos": está valorada por la mayoría de los encuestados con un 4, siendo su media de un 3,73 .

Tabla 14: Resultados de la afirmación 15, "Recomendaría este tipo de docencia a futuros alumnos"

\begin{tabular}{cccc}
\hline Media & Acuerdo & Neutral & Desacuerdo \\
\hline 3,73 & $65,67 \%$ & $22,39 \%$ & $11,94 \%$ \\
\hline
\end{tabular}

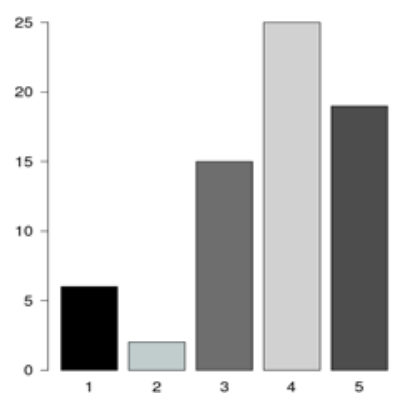

Figura 2: Resultados afirmación 15
Las dos afirmaciones restantes son las que tienen una menor valoración referente a la visión y utilidad de la asignatura, y son:

"Con esta metodología en clase, ha mejorado mi visión sobre la asignatura”: algo más de la mitad de los alumnos la han valorado con un 4 , pero un $10 \%$ aproximadamente con un 5 , por lo que la nota media ha bajado a 3,57.

Tabla 15: Resultados de la afirmación 14, “Con esta metodología en clase, ha mejorado mi visión sobre la asignatura”

\begin{tabular}{cccc}
\hline Media & Acuerdo & Neutral & Desacuerdo \\
\hline 3,57 & $62,69 \%$ & $26,87 \%$ & $10,44 \%$ \\
\hline
\end{tabular}

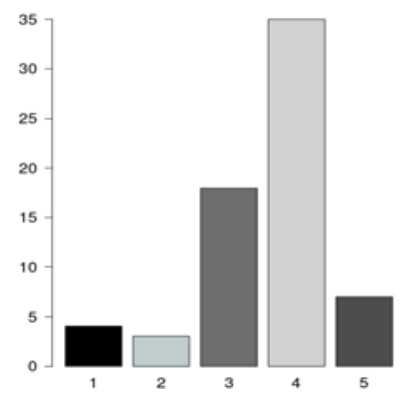

Figura 3: Resultados afirmación 14

Respecto a la afirmación "Las actividades realizadas en las clases inversas me han mostrado utilidades de la asignatura que sólo con teoría, no alcanzaría a apreciar”, han sido valoradas por 1 de cada 3 alumnos de manera neutral, lo que ha hecho que baje la media de la valoración.

Tabla 16: Resultados de la afirmación 16, “Las actividades realizadas en las clases inversas me han mostrado utilidades de la asignatura que sólo con teoría, no alcanzaría a apreciar”

\begin{tabular}{cccc}
\hline Media & Acuerdo & Neutral & Desacuerdo \\
\hline 3,28 & $43,28 \%$ & $37,31 \%$ & $19,41 \%$ \\
\hline
\end{tabular}

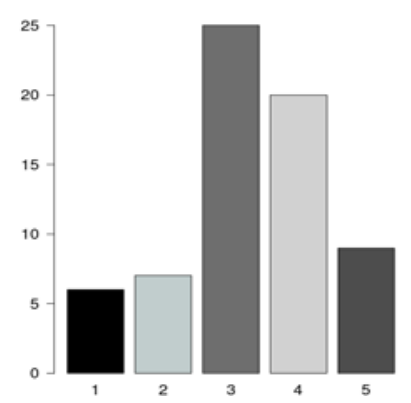

Figura 4: Resultados afirmación 16

\section{CONCLUSIONES}

En las respuestas de los alumnos puede apreciarse que les ha gustado utilizar herramientas como Kahoot! y YouTube, aunque aproximadamente uno de cada cinco considera que no le ha ayudado a estudiar. En el próximo curso se va a continuar con estas acciones. 
Hay un porcentaje muy bajo de alumnos que lee siempre la teoría, y es un hecho que se desea cambiar en los próximos cursos. Para ello, se realizará un Kahoot! al inicio de la primera clase de cada tema, comprobando así los conocimientos adquiridos. Dada la competitividad que han demostrado los alumnos al contestar este tipo de preguntas al cerrar los temas, se espera que se miren la teoría para poder contestar a las preguntas iniciales. Si se detecta que las respuestas no son buenas, cada una de estas pruebas será puntuable.

\section{AGRADECIMIENTOS}

Este trabajo ha sido financiado por la Escola Tècnica Superior d'Enginyeria Informàtica de la Universitat Politècnica de València.

\section{REFERENCIAS}

Bennett, B., Kern, J., Gudenrath, A., \& McIntosh, P. (2011). The flipped class revealed. The Daily Riff.

Bergmann, J., \& Sams, A. (2012). Flip your classroom: Reach every student in every class every day. International Society for Technology in Education.

Fidalgo-Blanco, A., Martinez-Nuñez, M., Borrás-Gene, O., \& Sanchez-Medina, J. J. (2017). Micro flip teaching-An innovative model to promote the active involvement of students. Computers in Human Behavior, 72, 713-723.

Fulton, K. (2012). Upside down and inside out: Flip your classroom to improve student learning. Learning \& Leading with Technology, 39(8), 12-17.

Gómez, A. G. (2017). Aprendizaje inverso y motivación en el aula universitaria. PULSO. Revista de Educación, (39), 199-218.
Barrera, A. G. (2013). El aula inversa: cambiando la respuesta a las necesidades de los estudiantes. Avances en Supervisión Educativa, (19).

Jinlei, Z., Ying, W., \& Baohui, Z. (2012). Introducing a New Teaching Model: Flipped Classroom [J]. Journal of Distance Education, 4(8), 46-51.

O'Flaherty, J., \& Phillips, C. (2015). The use of flipped classrooms in higher education: A scoping review. The Internet and Higher Education, 25, 85-95.

Prieto-Martín, A. (Ed.). (2017) Flipped learning: Aplicar el Modelo de Aprendizaje Inversa. Madrid, España: Narcea.

Sams, A., \& Bergmann, J. (2013). Flip your students' learning. Educational leadership, 70(6), 16-20.

Spencer, D., Wolf, D., \& Sams, A. (2011). Are you ready to flip? The Daily Riff.

Talbert, R. (2012). Inverted Classroom. Colleagues. 9(7), p. 14. Recuperado de http://scholarworks.gvsu.edu/colleagues/vol9/iss1/7 [Consulta: 15 de mayo de 2017].

Tedesco, J.C. (2010). La educación en el horizonte 2020. Madrid, España: Fundación Santillana.

Terrasa Barrena, S., \& Andreu García, G. (2015). Cambio a metodología de clase inversa en una asignatura obligatoria. In Actas del simposio-taller sobre estrategias y herramientas para el aprendizaje y la evaluación (pp. 32-37). Universitat Oberta La Salle.

Tourón, J., \& Santiago, R. (2015). El modelo Flipped Learning y el desarrollo del talento en la escuela Flilpped Learning model and the development of talent at school. Revista de Educación, 368, 196-231. 